\title{
Sexual Dysfunction and Neuroendocrine Correlates of Posttraumatic Stress Disorder inCombat Veterans: Preliminary Findings
}

\author{
Amy Lehrner ${ }^{1,2}$, Janine D. Flory ${ }^{1,2}$,Linda M. Bierer ${ }^{1}$, Iouri Makotkine ${ }^{2}$, Charles R. \\ Marmar $^{3}$, Rachel Yehuda $^{1,2}$
}

Author affiliations:

${ }^{1}$ James J. Peters Veterans Affairs Medical Center, Mental Health Care Center, Bronx, New York, USA

${ }^{2}$ Icahn School of Medicine at Mount Sinai, Department of Psychiatry, New York, New York, USA

${ }^{3}$ NYU Langone School of Medicine, New York, New York, USA

*Corresponding author

Amy Lehrner, $\mathrm{PhD}$

Mental Health Care Center, James J. Peters Veterans Affairs Medical Center 526 OOMH PTSD 116/A

130 West Kingsbridge Road

Bronx, New York 10468

Email: amy.lehrner@va.gov

FAX: (718) 741-4703

Phone: (718) 584-9000x3205

Keywords: sexual dysfunction, posttraumatic stress disorder, neurobiology, neuroendocrinology 


\begin{abstract}
Sexual dysfunction is not a symptom of PTSD but is a common clinical complaint in trauma survivors with this disorder. In that there are biological parallels in the neuroendocrine processes underlying both PTSD and sexual behavior, we conducted an exploratory investigation of the relationship of PTSD and related neuroendocrine indicators with sexual dysfunction in armed service veterans. Major Depressive Disorder, highly comorbid with PTSD and sexual dysfunction, was also assessed. In veterans with PTSD, sexual problems were associated with plasma DHEA and cortisol, urinary catecholamines, and glucocorticoid sensitivity, even when controlling for the effects of comorbid depression. In a subsample analysis, testosterone levels did not distinguish PTSD or sexual dysfunction, suggesting that sexual problems reported by veterans in this sample were not the result of organic disorder. PTSD did predict higher dihydrotestosterone (DHT) levels, which were associated with sexual problems. More detailed assessment of sexual dysfunction in biologically informed studies of PTSD is warranted to clarify the relationships of PTSD symptomatology and related neurobiology with sexual dysfunction.
\end{abstract}


Posttraumatic Stress Disorder (PTSD) can develop following exposure to a lifethreatening or horrifying experience, such as an accident, assault, natural disaster, terrorist attack, or combat. Symptoms of the disorder encompass intrusive memories, hyperarousal, avoidance of reminders, and changes in mood and cognitions (American Psychiatric Association, 2013). PTSD negatively impacts interpersonal relationships and is associated with marital separation, divorce, and intimate partner violence (e.g., Davidson et al., 1991; Glenn et al., 2002). PTSD has also been associated with sexual dysfunction (SD), which can include problems in desire, arousal, consummation, activity or satisfaction, in men and women (e.g., (Breyer et al., 2014; Yehuda, Lehrner, \& Rosenbaum, 2015). Rates of SD in PTSD range as high as $89 \%$ in male combat veterans, with up to a 30 -fold increase in risk for sexual problems such as erectile dysfunction, and the increased risk conferred by PTSD is seen in younger, as well as older, individuals (Yehuda, Lehrner, \& Rosenbaum, 2015). Hallmark symptoms of PTSD, such as intrusive memories and emotional numbing, may contribute to problems with sexual intimacy.

There is a large literature on the neuroendocrinology of PTSD (Zoladz \& Diamond, 2013), but a dearth of studies extending current knowledge of the biology of PTSD into research on associated SD. Yet there are notable biological parallels in the neuroendocrine processes underlying both PTSD and sexual behavior (Yehuda et al., 2015). The sympathetic nervous system and hypothalamic-pituitary-adrenal (HPA) axis are both activated by arousal, whether sexual or threat-induced, and it is possible that this confluence may be one mechanism whereby PTSD interferes with sexual functioning (Öznur, Akarsu, Karaahmetoğlu, \& Doruk, 2014). For example, catecholamine elevations are a central component of sexual response, but increased catecholamines such as norepinephrine have also been associated with PTSD diagnosis and 
symptom severity (Geracioti et al., 2001; Yehuda, Southwick, Giller, Ma, \& Mason, 1992).

Overly high levels of catecholamines may impair sexual performance directly or by triggering intrusive memories in persons with PTSD during sexual activity. Vivid trauma-related images during sexual activity have been reported in the literature and anecdotally in our outpatient PTSD clinic (Hirsch, 2009).

Despite the high comorbidity of PTSD and SD and the overlap in neuroendocrine processes relevant to both PTSD and sexual functioning, sexual dysfunction has been completely neglected in biological studies of PTSD. In this brief report we performed an exploratory study to investigate the relationship of SD with PTSD and with known neuroendocrine correlates of PTSD including cortisol and catecholamines (Zoladz \& Diamond, 2013), in veterans. Hormones relevant to sexual functioning (e.g., testosterone) were also analyzed in a subset of participants. Depression is an important potential confound in the emerging literature on PTSD and SD, given the high rate of comorbidity of PTSD and depression (roughly 50\% across diverse epidemiological samples) and the known association of depression with sexual dysfunction (Baldwin, 2001), and therefore Major Depressive Disorder (MDD) was also evaluated and included as a covariate.

\section{Methods}

Data from four studies of PTSD biomarkers in veterans were analyzed for this report. Participants in all studies: 1) had trauma exposure that met DSM-IV diagnostic criteria for PTSD (i.e., a "Criterion A" trauma), 2) provided blood (collected at $0800 \mathrm{~h}$ by routine venipuncture) and 24 h urine samples, 3) completed structured clinical interviews for Axis I diagnoses (e.g., MDD) and self-report measures, and 4) were assessed for PTSD by a clinical psychologist using the Clinician-Administered PTSD Scale for DSM-IV (CAPS). All study procedures were IRB 
approved, and all participants provided written, informed consent. Indicators of HPA axis function in blood (e.g., cortisol, DHEA, DHEA-S), glucocorticoid sensitivity (i.e., plasma cortisol suppression on the low-dose [0.5 mg] dexamethasone suppression test [DST]), and urinary catecholamines (epinephrine, norepinephrine, and dopamine) are analyzed in this report. Because of the association of age with sexual dysfunction (Feldman et al., 1994), all analyses include age as a covariate. When associated with the biological indicator of interest, BMI is an additional covariate in analyses of biological measures, which are log transformed when appropriate.

Two datasets were created from four separate studies of PTSD in veterans. The first dataset (Part I) includes data from an ongoing, cross-sectional study of Iraq and Afghanistan combat veterans who responded to referral, flyers, and letters seeking veterans for research participation. This report includes data from 170 male veterans with $(n=76)$ and without $(n=94)$ PTSD. Eligible participants had either a diagnosis of current PTSD related to their warzone experiences with a CAPS score $\geq 40$, or no PTSD diagnosis with a CAPS score $\leq 20$ and no history of PTSD. More details about inclusion/exclusion criteria and procedures are reported in (Yehuda et al., 2015a). Sex hormones (e.g., testosterone, estradiol, and dihydrotestosterone [DHT]) were assayed for two subgroups, veterans with PTSD who reported SD $(n=37)$ and veterans without PTSD with low levels of reported SD ( $n=24)$.

The second dataset (Part II) combines data from male treatment-seeking veterans of any era who participated in three treatment studies (two completed and one ongoing; $n=120$ ). These veterans were referred by their clinicians in an outpatient VA PTSD clinic to participate in a treatment study. Eligible participants had current PTSD with a CAPS $\geq 50$. More detailed inclusion/exclusion criteria and procedures are described in Yehuda et al., 2014; Yehuda et al., 
2015b. These two datasets were analyzed separately to minimize heterogeneity and missing data due to differences in the measures used, inclusion criteria, and recruitment strategies.

Sexual dysfunction was assessed by self-report items on multiple measures. The Beck Depression Inventory II (BDI; Beck, 1996) item 21 assesses "loss of interest in sex" in the past week with a 4-point response scale. The Social Adjustment Scale-Self Report (SAS; Weissman \& Bothwell, 1999) asks respondents living with a spouse or intimate how many times the respondent has had sex with a partner in the prior 2 weeks (5 point scale ranging from "more than twice a week" to "not at all in a month or longer"); and whether the respondent had "any problems during sexual relations during these last two weeks" (scale from none to no sexual relations). The Life Experiences Survey (LES; Sarason, Johnson, \& Siegel, 1978) item 16 asks participants to rate how negatively or positively "sexual difficulties" in the past year have impacted one's life ( 7 point scale) in the prior 6 months or 7 months to 1 year. The Expanded Health Symptom Checklist (HC; (Proctor et al., 1998) ranks problems that have been moderately severe or bothersome over the prior 30 days on a five point scale (none to almost every day). Item 35 is "loss of interest in sex," and item 36 is "difficulty achieving orgasm." The Symptom Checklist 90-Revised item 5 assesses loss of sexual interest in the prior 7 days using a 5-point scale (Derogatis \& Unger, 2010). Not all measures were administered across all studies; varied sample sizes reflect this.

\section{Results}

Demographic and Clinical Data

Table 1 presents demographic and clinical data for the two datasets. Both PTSD status and symptom severity were positively associated with SD such that: (1) veterans with PTSD were more likely to report SD than those without PTSD; and (2) among those with PTSD the 
severity of symptoms was also associated with SD. These associations remained significant even when controlling for MDD. In Part I, veterans with PTSD were more likely to report a loss of interest in sex over the previous two weeks on the BDI than veterans without PTSD $\left(\mathrm{F}_{2,167}=70.11, \mathrm{p}<.0001\right)$. PTSD was also highly comorbid with depression, as expected $(57.9 \%$ with PTSD were also currently experiencing a Major Depressive Episode). However, the association of sexual problems with PTSD does not appear to be driven solely by depression. PTSD remained a significant predictor when the presence of a current Major Depressive Episode, coded as a dichotomous variable, was included as a (significant) covariate. Among those with PTSD, SD was also positively associated with symptom severity, even when controlling for depression, in a hierarchical linear regression analysis (see Table 2a). Association of neuroendocrine markers with sexual problems

In Part I, there were no associations of neuroendocrine markers with both PTSD status or symptom severity and SD. However, among veterans with PTSD, loss of interest in sex in the past 7 days was associated with the adrenal androgen DHEA $\left(\beta=-.316, t_{72}=-2.364, p=.021\right)$ and plasma cortisol $\left(\beta=-.378, t_{72}=-3.344, \mathrm{p}=.001\right)$ in separate linear regression analyses (see Table 2b). With respect to glucocorticoid sensitivity, cortisol decline following ingestion of dexamethasone was negatively associated with loss of interest in sex in the past 7 days $(\beta=-.314$, $\left.t_{67}=-2.565, \mathrm{p}=.013\right)$. These indicators remained significant when current depression was included as a predictor. Regarding urinary catecholamines, a higher NE/cortisol ratio, also known to be elevated in PTSD (Mason, Giller, Kosten, \& Harkness, 1988) and reflecting the dual influences of heightened physiological arousal (NE) and attenuated containment of such arousal (cortisol), was correlated with the presence of problems during sexual relations $(\mathrm{r}=.382, \mathrm{p}=.028, \mathrm{df}=31)$. 
The NE/cortisol ratio specifically predicted intrusive symptoms on the CAPS $\left(\beta=.216, t_{89}=2.04\right.$, $\mathrm{p}=.044)$.

In Part II, urinary NE was associated with difficulty achieving orgasm in a regression analysis $\left(\beta=.340, t_{39}=2.34, p=.024\right)$, a relationship that was unchanged when current SSRI use was included as a (nonsignificant) covariate. Urinary NE also explained a significant proportion of variance in difficulty achieving $\operatorname{orgasm}\left(\mathrm{R}^{2}=.22, \mathrm{~F}_{3,39}=3.66, \mathrm{p}=.02\right)$. Association of PTSD and sexual dysfunction with sex hormones

All sex hormones were within the normal range, suggesting that the reported SD is not the result of organic endocrine disorder. Furthermore, there was no group difference in testosterone levels. However, PTSD was associated with higher levels of the androgen DHT (PTSD: $\left.\beta=.361, t_{60}=2.973, \mathrm{p}=.004\right)$, and in separate regression analyses, DHT was associated with loss of interest in $\operatorname{sex}\left(\beta=.377, t_{60}=3.126, p=.003\right)$, frequency of $\operatorname{sex}\left(\beta=.445, t_{46}=3.293\right.$, $\mathrm{p}=.002)$, and sex difficulties in the past year $(\mathrm{OR}=.993$, Wald $=6.80, \mathrm{p}=.009)$. To examine whether DHT was associated with additional variance after accounting for PTSD, the variables were entered in separate steps in the same regression. PTSD was a significant predictor of the SD outcomes above, but DHT was not (PTSD: $0.004<\mathrm{p} \leq .0001$; DHT: $0.055<\mathrm{p}<.127)$. The addition of DHT did not result in a significantly greater adjusted R squared $\left(\left(<.014 \Delta \mathrm{AR}^{2}<.045\right)\right.$. Lower levels of estradiol were also associated with loss of interest in $\operatorname{sex}\left(\beta=-.314, \mathrm{t}_{60}=-2.467\right.$, $\mathrm{p}=.017)$ and less frequent $\operatorname{sex}\left(\beta=-.432, \mathrm{t}_{46}=-3.085, \mathrm{p}=.004\right)$, but estradiol was not associated with PTSD.

\section{Discussion}

These findings indicate that known neuroendocrine correlates of PTSD are associated with SD, and these relationships do not appear to be artifacts of current Major Depressive 
Disorder (MDD). In veterans with PTSD, loss of sexual interest was associated with lower levels of plasma DHEA and ambient cortisol and an attenuated response to the DST, findings which remained significant when depression was included in analyses. Heightened catecholamines as reflected by the NE/cortisol ratio were also associated with problems during sexual relations and specifically with intrusive symptoms. In a separate study of treatment seeking veterans with PTSD, urinary NE was also associated with SD, in this case difficulty achieving orgasm.

PTSD has been associated with relatively higher glucocorticoid sensitivity, higher levels of DHEA and catecholamines, and lower levels of cortisol, whereas depression has been associated with lower glucocorticoid sensitivity and higher cortisol (Naughton, Dinan, \& Scott, 2013). Therefore, among the participating male veterans with PTSD in these studies, neuroendocrine markers consistent with both PTSD and depressive symptomatology were associated with SD, even when controlling for the effects of a comorbid MDD diagnosis. For example, lower (rather than higher) glucocorticoid sensitivity on the DST was associated with SD among veterans with PTSD. It should be noted that diagnosing comorbid depression is complicated by the fact that many DSM diagnostic criteria for PTSD are also symptoms of depression (e.g., anhedonia), and DSM 5 revisions further emphasize alterations in mood, such as depression, guilt, and shame. Such symptoms may be particularly relevant for sexual functioning in PTSD. It is possible that different symptom presentations and neurobiological signatures may associate with distinct aspects of SD in those with PTSD. For example, it may be that depressive symptoms and numbing, and reduced glucocorticoid sensitivity, are associated with a loss of libido; whereas re-experiencing and hyperarousal, and heightened catecholamine activity, are associated with difficulty in performance. In conjunction with anecdotal reports of 
intrusive symptoms during sexual activity, the association of catecholamines (i.e., urinary NE

and the NE/cortisol ratio) with sexual problems (including difficulty achieving orgasm) and

intrusive symptoms in this study lends support to the hypothesis that PTSD related sympathetic

nervous system arousal may interfere with sexual performance. Further research is needed to

investigate the potential mediating influence of NE on PTSD related SD.

Regarding sex hormones, testosterone levels did not distinguish PTSD or SD in a subsample analysis, suggesting that sexual problems reported by veterans in this sample were not the result of organic disorder. However, higher levels of the androgen DHT was associated with multiple indicators of SD, including loss of interest and problems with performance, as well as with PTSD. DHT is generally associated with good sexual function, but research on the effects of supplementation with DHT has been mixed. It has also been hypothesized that aromatization of testosterone is important for sexual function, and that selective estrogen deficiency, such as can be induced by high levels of DHT, may lead to impaired sexual function (Finkelstein et al., 2013; Sartorius, Ly, and Handelsman, 2014). A small study in healthy middle-aged men found that transdermal DHT administration produced a statistically significant but subclinical reduction in sexual desire (Sartorius, Ly, and Handelsman, 2014). Our findings of lower estradiol in association with SD are consistent with this hypothesis.

These findings were based on data gathered for other purposes, and as such all analyses are post hoc. Reported analyses rely on single items to assess aspects of sexual functioning, and while these items were correlated across measures, the observations reported here represent preliminary findings. The current study was also limited to male veterans. Biological parallels in the neuroendocrine processes underlying both PTSD and sexual behavior are similar in both men and women (Yehuda et al., 2015), but there are sex specific aspects of SD, women's hormonal 
milieu is different from men's, and women's trauma exposure is often more interpersonal and sometimes sexual in nature (e.g., rape), with potentially specific SD sequelae.

Despite the limitations, these initial findings of neuroendocrine correlates of SD in PTSD are suggestive of potential biological mechanisms through which PTSD may lead to impaired sexual function, Future biologically informed studies of sexual dysfunction associated with PTSD should utilize validated, gender specific measures of sexual dysfunction, comprehensive assessments of trauma type and PTSD symptoms, and relevant sex hormone assays in adequately powered samples of men and women. Qualitative interview data may also help characterize SD in PTSD, identifying specific phenotypes and generating hypotheses for further investigation. The current literature on SD in PTSD suggests a growing recognition of the importance of this domain of functioning for PTSD related illness, recovery and quality of life (Yehuda, Lehrner, \& Rosenbaum, 2015). However, few studies include comprehensive assessments of both PTSD symptomatology and SD, and none have been identified that take a biologically informed approach. Such biologically-informed research on sexual function in PTSD is highly clinically relevant, and may ultimately inform the development of either psychotherapeutic and pharmacologic treatments or augmentations. 


\section{References}

American Psychiatric Association. (2013). Diagnostic and statistical manual of mental disorders (5th ed.). Washington, DC: Author.

Baldwin, D. S. (2001). Depression and sexual dysfunction. British Medical Bulletin, 57(1), 8199. doi: $10.1093 / \mathrm{bmb} / 57.1 .81$

Beck, A.T., Steer, R.A., \& Brown, G.K. (1996). Manual for the Beck Depression Inventory-II. San Antonio, TX: Psychological Corporation.

Breyer, B. N., Cohen, B. E., Bertenthal, D., Rosen, R. C., Neylan, T. C., \& Seal, K. H. (2014). Sexual dysfunction in male Iraq and Afghanistan war veterans: association with posttraumatic stress disorder and other combat-related mental health disorders: a population-based cohort study. J Sex Med, 11(1), 75-83. doi: 10.1111/jsm.12201

Davidson, J. R., Hughes, D., Blazer, D. G., \& George, L. K. (1991). Post-traumatic stress disorder in the community: an epidemiological study. Psychological medicine, 21(03), 713-721.

Derogatis, L. R., \& Unger, R. (2010). Symptom checklist-90-revised. Corsini encyclopedia of psychology.

Feldman, H. A., Goldstein, I., Hatzichristou, D. G., Krane, R. J., \& McKinlay, J. B. (1994). Impotence and its medical and psychosocial correlates: results of the Massachusetts Male Aging Study. The Journal of Urology, 151(1), 54-61.

Finkelstein, J. S., Lee, H., Burnett-Bowie, S. A. M., Pallais, J. C., Yu, E. W., Borges, L. F., ... \& Leder, B. Z. (2013). Gonadal steroids and body composition, strength, and sexual function in men. New England Journal of Medicine, 369(11), 1011-1022. 
Geracioti, T. D., Jr., Baker, D. G., Ekhator, N. N., West, S. A., Hill, K. K., Bruce, A. B., . . . Kasckow, J. W. (2001). CSF norepinephrine concentrations in posttraumatic stress disorder. American Journal of Psychiatry, 158(8), 1227-1230.

Glenn, D. M., Beckham, J. C., Feldman, M. E., Kirby, A. C., Hertzberg, M. A., \& Moore, S. D. (2002). Violence and hostility among families of Vietnam veterans with combat-related posttraumatic stress disorder. Violence and victims, 17(4), 473-489.

Hirsch, K. A. (2009). Sexual dysfunction in male operation enduring freedom/operation Iraqi freedom patients with severe post-traumatic stress disorder. Military Medicine, 174, 520522.

Mason, J. W., Giller, E. L., Kosten, T. R., \& Harkness, L. (1988). Elevation of urinary norepinephrine/cortisol ratio in posttraumatic stress disorder. J Nerv Ment Dis, 176(8), 498-502.

Naughton, M., Dinan, T., \& Scott, L. (2013). Corticotropin-releasing hormone and the hypothalamic-pituitary-adrenal axis in psychiatric disease. Handbook of clinical neurology, 124, 69-91.

Öznur, T., Akarsu, S., Karaahmetoğlu, B., \& Doruk, A. (2014). A rare symptom in posttraumatic stress disorder: Spontaneous ejaculation. The American journal of case reports, 15, 69.

Proctor, S. P., Heeren, T., White, R. F., Wolfe, J., Borgos, M. S., Davis, J. D., . . Ozonoff, D. (1998). Health status of Persian Gulf War veterans: self-reported symptoms, environmental exposures and the effect of stress. Int J Epidemiol, 27(6), 1000-1010.

Sarason, I. G., Johnson, J. H., \& Siegel, J. M. (1978). Assessing the impact of life changes: development of the Life Experiences Survey. Journal of consulting and clinical psychology, 46(5), 932 
Sartorius, G. A., Ly, L. P., \& Handelsman, D. J. (2014). Male Sexual Function Can Be Maintained Without Aromatization: Randomized Placebo-Controlled Trial of Dihydrotestosterone (DHT) in Healthy, Older Men for 24 Months. The journal of sexual medicine, 11(10), 2562-2570.

Serretti, A., \& Chiesa, A. (2009). Treatment-emergent sexual dysfunction related to antidepressants: a meta-analysis. Journal of clinical psychopharmacology, 29(3), 259266.

Southwick, S.M., Morgan, C.A., 3rd, Charney, D.S., High, J.R. (1999). Yohimbine use in a natural setting: effects on posttraumatic stress disorder. Biological Psychiatry, 46(3):4424.

Weissman, M.M., Bothwell, S. (1976). Assessment of Social Adjustment by Patient Self-Report. Archives of General Psychiatry, 33(9),1111-1115.

Yehuda, R., Bierer, L. M., Pratchett, L. C., Lehrner, A., Koch, E. C., Van Manen, J. A., ... \& Hildebrandt, T. (2015). Cortisol augmentation of a psychological treatment for warfighters with posttraumatic stress disorder: Randomized trial showing improved treatment retention and outcome. Psychoneuroendocrinology, 51, 589-597.

Yehuda, R., Flory, J.D., Bierer, L.M., Henn-Haase, C., Lehrner, A., Desarnaud, F., Makotkine, I., Daskalakis, N.P., Marmar, C.R., Meaney, M.J. (2015a). Lower methylation of glucocorticoid receptor gene promoter $1 \mathrm{~F}$ in peripheral blood of veterans with posttraumatic stress disorder. Biol Psychiatry. 77(4),356-64.

Yehuda, R., Lehrner, A., \& Rosenbaum, T. (2015). PTSD and Sexual Dysfunction in Men and Women. Journal of Sexual Medicine, DOI: 10.1111/jsm.12856. 
Yehuda, R., Pratchett, L. C., Elmes, M. W., Lehrner, A., Daskalakis, N. P., Koch, E., ... \& Bierer, L. M. (2014). Glucocorticoid-related predictors and correlates of post-traumatic stress disorder treatment response in combat veterans. Interface focus, 4(5), 20140048.

Yehuda, R., Southwick, S., Giller, E. L., Ma, X., \& Mason, J. W. (1992). Urinary catecholamine excretion and severity of PTSD symptoms in Vietnam combat veterans. J Nerv Ment Dis, 180(5), 321-325.

Zoladz, P. R., \& Diamond, D. M. (2013). Current status on behavioral and biological markers of PTSD: a search for clarity in a conflicting literature. Neuroscience \& Biobehavioral Reviews, 37(5), 860-895. 
Table 1. Demographics and Clinical Data

\begin{tabular}{|c|c|c|c|}
\hline & \multicolumn{2}{|c|}{ Study A (n=170) } & Study B $(n=137)$ \\
\hline & \multicolumn{2}{|c|}{$M \pm S D$ or $n(\%)$} & $M \pm S D$ or $n(\%)$ \\
\hline Age & \multicolumn{2}{|c|}{$32.57 \pm 8.11$} & $43.23 \pm 13.72$ \\
\hline \multicolumn{4}{|l|}{ Race } \\
\hline Black & \multicolumn{2}{|c|}{$49(28 \%)$} & $74(54 \%)$ \\
\hline White & \multicolumn{2}{|c|}{$71(40.6 \%)$} & $48(35 \%)$ \\
\hline Asian & \multicolumn{2}{|c|}{$10(5.7 \%)$} & $2(1.5 \%)$ \\
\hline Other & \multicolumn{2}{|c|}{$20(11.4 \%)$} & $5(3.6 \%)$ \\
\hline Ethnicity & \multicolumn{2}{|c|}{ Hispanic: 64 (36.6\%) } & Hispanic: 67 (48.9\%) \\
\hline \multicolumn{4}{|l|}{ Conflict } \\
\hline OEF/OIF/OND & \multicolumn{2}{|c|}{$175(100 \%)$} & $96(70.1 \%)$ \\
\hline Vietnam & \multicolumn{2}{|c|}{--} & $35(25.5 \%)$ \\
\hline Gulf War/Other & \multicolumn{2}{|c|}{--} & $6(4.4 \%)$ \\
\hline \multirow[t]{2}{*}{ CAPS total score } & $\operatorname{PTSD}(+)$ & PTSD (-) & $78.35 \pm 19.19$ \\
\hline & $69.18 \pm 16.87$ & $3.73 \pm 5.19$ & \\
\hline \multirow{2}{*}{$\begin{array}{l}\text { Major Depressive } \\
\text { Disorder }\end{array}$} & PTSD (+) & PTSD (-) & $51(37.2 \%)$ \\
\hline & $44(57.9 \%)$ & $3(3.2 \%)$ & \\
\hline
\end{tabular}

Figure Legend. $\mathrm{OEF} / \mathrm{OIF} / \mathrm{OND}=$ Operation Enduring Freedom, Operation Iraqi Freedom, Operation New Dawn; CAPS = Clinician-Administered PTSD Scale for DSM-IV; Major Depressive Disorder=presence of current DSM-IV diagnosis of Major Depressive Disorder. 
Table 2a. Regression table of PTSD symptom severity, Major Depressive Disorder, and Loss of Sexual Interest

\begin{tabular}{|c|c|c|c|}
\hline Predictors & Standardized Coefficient $(\beta)$ & $\mathrm{p}$ & $\mathrm{R}^{2}$ \\
\hline Step 1 & & & .002 \\
\hline Age & -.042 & .718 & \\
\hline Step 2 & & & .345 \\
\hline Age & -.010 & .930 & \\
\hline MDD & .344 & .003 & \\
\hline Step3 & & & .432 \\
\hline Age & -.028 & .792 & \\
\hline MDD & .242 & .038 & \\
\hline PTSD symptom severity & .279 & .017 & \\
\hline
\end{tabular}


Table 2b.Regression Table of Neuroendocrine Indicators with Loss of Sexual Interest in Veterans with PTSD

\begin{tabular}{|c|c|c|c|c|c|c|c|c|c|c|}
\hline & & \multicolumn{3}{|c|}{ Step 1} & \multicolumn{3}{|c|}{ Step 2} & \multicolumn{3}{|l|}{ Step 3} \\
\hline & & $\begin{array}{l}\text { Standardized } \\
\text { Coefficient }(\beta)\end{array}$ & $\mathrm{p}$ & $\begin{array}{l}\text { Adjusted } \\
\mathrm{R}^{2}\end{array}$ & $\begin{array}{l}\text { Standardized } \\
\text { Coefficient }(\beta)\end{array}$ & $\mathrm{p}$ & $\begin{array}{l}\text { Adjusted } \\
\mathrm{R}^{2}\end{array}$ & $\begin{array}{l}\text { Standardized } \\
\text { Coefficient }(\beta)\end{array}$ & $\mathrm{p}$ & $\begin{array}{l}\text { Adjusted } \\
\mathrm{R}^{2} \\
\end{array}$ \\
\hline DHEA & $\begin{array}{l}\text { constant } \\
\text { Age } \\
\text { BMI } \\
\text { MDD } \\
\text { DHEA }\end{array}$ & $\begin{array}{l} \\
.045 \\
.029\end{array}$ & $\begin{array}{l}.707 \\
.907\end{array}$ & -.025 & $\begin{array}{l}- \\
-.114 \\
.046 \\
-.316 \\
\end{array}$ & $\begin{array}{l}.011 \\
.398 \\
.690 \\
.021 \\
\end{array}$ & .038 & $\begin{array}{l}- \\
-.061 \\
.068 \\
.382 \\
-.290 \\
\end{array}$ & $\begin{array}{l}.061 \\
.624 \\
.530 \\
.001 \\
.022 \\
\end{array}$ & .176 \\
\hline CORTISOL & $\begin{array}{l}\text { constant } \\
\text { Age } \\
\text { BMI } \\
\text { MDD } \\
\text { Cortisol }\end{array}$ & $\begin{array}{l}- \\
.045 \\
.029\end{array}$ & $\begin{array}{l}.195 \\
.707 \\
.807\end{array}$ & -.025 & $\begin{array}{l}- \\
-.016 \\
.019 \\
- \\
-.378\end{array}$ & $\begin{array}{l}.007 \\
.889 \\
.868\end{array}$ & .105 & $\begin{array}{l}- \\
.030 \\
.040 \\
.327 \\
-.299\end{array}$ & $\begin{array}{l}.089 \\
.780 \\
.705 \\
.004 \\
.008\end{array}$ & .196 \\
\hline $\begin{array}{l}\text { CORTISOL } \\
\text { DECLINE }\end{array}$ & $\begin{array}{l}\text { Constant } \\
\text { Age } \\
\text { BMI } \\
\text { MDD } \\
\text { CORT } \\
\text { decline }\end{array}$ & $\begin{array}{l}- \\
.057 \\
-.016\end{array}$ & $\begin{array}{l}.177 \\
.645 \\
.898\end{array}$ & -.027 & $\begin{array}{l}- \\
-.014 \\
-.045 \\
\\
-.314\end{array}$ & $\begin{array}{l}.019 \\
.908 \\
.709\end{array}$ & .054 & $\begin{array}{l}- \\
.024 \\
-.007 \\
.361 \\
-.270\end{array}$ & $\begin{array}{l}.124 \\
.833 \\
.948 \\
.002 \\
\\
.023\end{array}$ & .173 \\
\hline
\end{tabular}

Note: the dependent variable was loss of interest in sex in prior 7 days. DHEA log transformed.BMI: Body mass index; MDD: current Major

Depressive Episode; Cortisol: plasma cortisol; Cortisol Decline: cortisol decline following low-dose DST. 


\section{Acknowledgements}

Parent studies for the data included in this report were funded by the Lightfighter Trust (LFT2009-02-1) and the US Department of Defense (DOD W81XWH-06-0032, DOD W81XWH-10-2-0072, DOD W81XWH-09-2-0044). 